\title{
Notch1 inactivation promotes invasion and metastasis of nasopharyngeal carcinoma cells partly through Slug activation
}

\author{
J. S. AN ${ }^{1, *}$, Y. S. RHO ${ }^{2, *}$, J. H. MOON ${ }^{1}$, Y. C. LIM $^{1, *}$ \\ ${ }^{1}$ Department of Otorhinolaryngology - Head and Neck Surgery, Research Institute of Medical Science, Konkuk University School of Medicine, \\ Seoul, Korea; ${ }^{2}$ Department of Otorhinolaryngology - Head and Neck Surgery, Hallym University College of Medicine, Seoul, Korea \\ ${ }^{*}$ Correspondence: yclim69@hanmail.net \\ "Contributed equally to this work.
}

Received March 13, 2019 / Accepted June 19, 2019

\begin{abstract}
Nasopharyngeal carcinoma (NPC) is a rare form of the head and neck cancer of the epithelial lining of the nasopharynx and exhibits the highest metastatic rate among head and neck cancers. Underlying mechanisms of metastasis remain largely unknown. Here, we explored whether Notch1 affects the invasion and metastasis of NPC cells. In vitro migration and invasion capacities were evaluated after the knockdown of Notch1 expression in NPC cells. To investigate the role of Notch1 in in vivo metastasis, we examined the metastatic ability to the lungs following administration of cancer cells via mouse tail vein. The expression of epithelial-mesenchymal transition (EMT) markers associated with Notch1-mediated metastasis was investigated, and their roles in metastasis and relationship with Notch1 expression were investigated. Suppression of Notch1 expression increased the ability of NPC cells to invade Matrigel in vitro. Knockdown of Notch1 expression in NPC cells resulted in extensive lung metastasis in a mouse model and increased the mRNA expression of Slug in NPC cells. Slugspecific RNA interference resulted in the loss of the metastatic and invasion capacities in Notch1-suppressed NPC cells. These findings show that Notch1 has a significant suppressive role in the regulation of metastasis in NPCs, suggestive of its prudent use in clinical trials.
\end{abstract}

Key words: head and neck cancer, nasopharyngeal carcinoma, metastasis, Notch1, Slug

Nasopharyngeal carcinoma (NPC) is a non-lymphomatous, squamous-cell carcinoma that occurs in the epithelial lining of the nasopharynx [1]. NPC is a rare disease that is highly prevalent in Southeast Asia, especially in southern China with an incidence rate of $15-50$ per 100,000 people [2]. NPC exhibits the highest metastatic rate among head and neck cancers, and $20 \%$ of patients show distant metastasis, most often in the lungs, at the time of diagnosis $[3,4]$. A significant proportion of patients experiences tumor relapse after extensive conventional therapy, including chemotherapy and radiotherapy $[3,4]$. The molecular mechanisms underlying NPC metastasis are yet incompletely understood. Therefore, the discovery of the determinant associated with metastasis that may serve as a therapeutic target is desirable.

In mammals, the Notch signaling pathway is activated through the communication between four transmembrane Notch receptors (Notch1, Notch2, Notch and Notch4) and five membrane-bound ligands (DLL1, DLL3, DLL4, JAG1 and JAG2) $[5,6]$. This pathway classically functions in development and differentiation but also plays an important role in cancer. Notch activity affects differentiation, proliferation and apoptotic programs, serving as a developmental tool to influence organ formation and morphogenesis [7]. In cancer, the role of Notch pathway remains controversial. Notch receptors have been identified as oncogenes in multiple tumors, including leukemia, breast, colorectal, cervical, lung and oral squamous cell carcinomas. On the contrary, some studies have suggested their tumor suppressor role in breast, prostate, liver, glioblastoma, lung, bladder, skin and head and neck squamous cell carcinoma (HNSCC) $[8,9]$. In addition, Notch signaling positively regulates the epithelial-mesenchymal transition (EMT) and invasion of breast cancer cells. Notch was also shown to suppress angiogenesis and progression of hepatic metastases in neuroblastoma [10]. Thus, the Notch signaling pathway may exhibit oncogenic or tumor suppressor functions depending on the cellular nature. However, the role of Notch1 in the metastasis of NPC remains largely unknown. Considering that Notch pathway has a tumor suppressive role in several types of squamous carcinoma, we hypothesize that it may act as a tumor 
suppressor in NPC. In the present study, we demonstrate that the inhibition of Notch 1 expression causes an increase in the invasion and metastasis of NPC cells via downregulation of Slug expression, suggestive of the potential adverse implications of Notch inhibition.

\section{Material and methods}

Cell lines and culture. Two NPC cell lines, HNE1 and HONE1, were obtained from Professor CH Kim (Ajou University, Korea). HNE1 cells were cultured in Dulbecco's modified Eagle's medium (DMEM) low glucose with 10\% fetal bovine serum (FBS), while HONE1 cells were cultured in Roswell Park Memorial Institute-1640 (RPMI-1640) with $10 \%$ FBS. All cell lines were maintained at $37^{\circ} \mathrm{C}$ in $5 \% \mathrm{CO}_{2}$.

Knockdown of Notch 1 gene. Lentiviral packaging vectors (pCMV-VSVG and pCMV-delta R8.2, obtained from the professor HK Kim, Korea University, Korea) and scramble vector (pLKO.1-puro Vector, Sigma, St. Louis, MO, USA) or transfection vector (pLKO.1-puro-shNotch1 Vector, Sigma, St. Louis, MO, USA) were co-transfected in 293T cells using calcium phosphate precipitation method. Calcium phosphate precipitation was carried out with the CalPhos ${ }^{\mathrm{TM}}$ Mammalian Transfection Kit (Takara, Mountain View, CA, USA). Infectious lentiviruses were harvested at 48 and $72 \mathrm{~h}$ post-transfection and filtered through $0.45 \mu \mathrm{m}$ filters (Millipore, Billerica, MA, USA). To infect cells using lentivirus, NPC cells were seeded on a $100 \mathrm{~mm}$ dish at $60 \%$ confluence the day before infection. The following day, the medium was replaced with $10 \mathrm{ml}$ fresh medium containing lentivirus in the presence of $8 \mu \mathrm{g} / \mathrm{ml}$ of polybrene (Invitrogen, Carlsbad, CA, USA). After $6 \mathrm{~h}$, the medium was replaced with fresh medium. Cells were verified for the knockdown of Notch1 expression with western blotting. The short-hairpin RNA (shRNA) sequence used for Notch1 knockdown was as follows: shNotch1, 5'-CCGGCCGGGACATCACGGATCATATCTCGAGATATGATCCGTGATGTCCCGGTTTTTG-3' (Sigma, Sigma, St. Louis, MO, USA)

Knockdown of SLUG gene. HNE1 and HONE1 cells transduced with shNotch1 were transfected with the siSlug-pcDNA or negative control pcDNA using TransITLT1 Reagent (Mirus, Madison, WI, USA) in Opti-MEM (GIBCO, Grand Island, NY, USA) supplemented with 10\% FBS. After $48 \mathrm{~h}$ of incubation, the medium was replaced with $10 \mathrm{ml}$ of fresh culture medium. Cells were verified for the knockdown of Notch1 expression with western blotting. The sequence used for SLUG knockdown was as follows: siSlug, 5'-CAGACCCATTCTGATGTAAAG-3' (Addgene, Watertown, MA, USA).

Reverse transcriptase-polymerase chain reaction (RT-PCR). The RNA was isolated with Trizol Reagent (Invitrogen, Carlsbad, USA). cDNA was constructed by using ReverTraAce qPCR RT Master Mix with gDNA Remover (Toyobo, Tokyo, Japan). Amplification was performed with 25 ng cDNA, specific primer pairs and EmeraldAmp PCR
Master Mix (Takara, Mountain View, CA, USA). The amplification was done for $28 \mathrm{cycles}\left(95^{\circ} \mathrm{C} 1 \mathrm{~min}, 55^{\circ} \mathrm{C} 1 \mathrm{~min}, 72^{\circ} \mathrm{C}\right.$ $1 \mathrm{~min})$. PCR products were separated by $1.5 \%$ agarose gel electrophoresis and detected under ultraviolet light (Bio-Rad, Hercules, CA, USA). cDNA synthesis was monitored by PCR with GAPDH-primers. Primers used for RT-PCR were as follows: Twist F: 5' - CAG TCT TAC GAG GAG CTG CA-3', Twist R: 5'- TCT TGC TCA GCT TGT CCG AG -3', Slug F: 5'- CTC CAT GCC TGT CAT ACC AC -3', slug R: 5'- GGA AAG AGG AGA GAG GCC AT -3', Snail F: 5' - GCT GAC CTC CCT GTC AGA TG-3', Snail R: 5' - TGC TTG GGC ACT TGG CCC AA -3', GAPDH F: 5'- GGA GCG AGA TCC CTC CAA AA-3', GAPDH R: 5' - CAC ACC CAT GAC GAA CAT GG -3'

Western blot analysis. Cells were lysed with radioimmunoprecipitation assay (RIPA) buffer (50 mM Tris- $\mathrm{HCl} \mathrm{pH} \mathrm{7.4,}$ $150 \mathrm{mM} \mathrm{NaCl}, 1 \% \mathrm{NP}-40,0.1 \%$ sodium deoxycholate, $1 \mathrm{mM}$ ethylenediaminetetraacetic acid (EDTA), and $1 \mathrm{mM}$ phenylmethylsulphonyl fluoride) supplemented with protease inhibitor (Roche, Indianapolis, IN, USA) and phosphatase inhibitor ( $1 \mathrm{mM} \mathrm{NaF}$ and $1 \mathrm{mM} \mathrm{Na}_{3} \mathrm{VO}_{4}$ ). Protein concentration was assessed with the bicinchoninic acid (BCA) assay kit (Thermo Scientific, Rockford, USA). Proteins were separated on $8 \%$ or $10 \%$ acrylamide gels and transferred onto a polyvinylidene fluoride membrane (Millipore, Billerica, MA, USA). The membrane was blocked with 5\% non-fat dry milk in Tris-buffered saline containing $0.1 \%$ Tween-20 (TBST, Biosesang, Gyeonggi-do, Korea) at room temperature for $1 \mathrm{~h}$. The membrane was subsequently incubated with primary antibodies overnight at $4{ }^{\circ} \mathrm{C}$. The membrane was washed twice with TBST and incubated with secondary antibodies at room temperature for $1 \mathrm{~h}$. The protein bands were developed with enhanced chemiluminescence reagent (Thermo Scientific, Rockford, USA). Antibodies used for western blotting were as follows: NICD, $\beta$-actin (Cell Signaling Technology, Beverly, USA), Slug, matrix metalloproteinase (MMP)-2, MMP-9 (Santa Cruz Biotechnology, La Jolla, USA), and MMP-13 (Chemicon International, Billerica, USA) antibodies.

Wound-healing assay. Cells were seeded at the density of $10^{6}$ cells/well on a six-well plate. Cell monolayers were wounded using a plastic tip. Cells were washed twice with PBS to remove cell debris and incubated with fresh medium $(3 \mathrm{ml})$. The cells that migrated into the wound area were imaged and the average distance of the migrating cells was measured at time 0 and after $18 \mathrm{~h}$. The average distance per field was measured under $\times 100$ objective magnification from three microscopic fields.

Migration and invasion assay. The migration ability of the cells was assayed using a transwell $(8 \mu \mathrm{m}$ pore size, Corning Costar, Bedford, USA), while their invasion ability was evaluated with Transwell ( $8 \mu \mathrm{m}$ pore size, Corning Costar). Transfected cells were resuspended in $500 \mu \mathrm{l}$ of medium supplemented with $0.1 \%$ FBS and seeded onto the upper chamber at a concentration of $1.5 \times 10^{4}$ cells/well. Cells were incubated at 
$37^{\circ} \mathrm{C}$ in $5 \% \mathrm{CO}_{2}$. After incubation, an area devoid of cells was created at time $0 \mathrm{~h}$ by scraping a monolayer of the indicated cells, followed by overnight incubation of the cells in the absence of serum. The cells that spread on the lower surface of the membrane were fixed with $4 \%$ paraformaldehyde and stained with crystal violet, while those on the upper surface were removed with a cotton swab. The average number of cells per field was counted under $\times 100$ objective magnification from five microscopic fields.

In vivo metastasis. HNE1 and HONE1 cells transduced with shNotch 1 were intravenously injected $\left(10^{6}\right.$ cells/animal $)$ into the tails of 8 -week-old female BALB/c nude mice using a 22 -gauge needle. The mice were sacrificed 21 weeks after the injection of cells. The entire lung was excised, fixed and embedded in paraffin. The tissues were sectioned for hematoxylin and eosin $(\mathrm{H} \& \mathrm{E})$ staining. All animal studies were approved by the Institutional Animal Care and Use Committee of the Konkuk University.

Luciferase assay. HNE1 and HONE1 cells transduced with shNotch 1 were seeded onto 12 well plates $\left(6 \times 10^{4}\right.$ cells/ well). Cells were co-transfected with the indicated SlugMyc vector (pGL3-SlugMyc-Luc, Addgene, Watertown, MA, USA), negative control (pGL3-Basic), or positive control (pGL3-control) and the Renilla luciferase expression vector (pRL-SV40) using Lipofectamine Reagent (Invitrogen, Carlsbad, USA) in Opti-MEM (GIBCO, Grand Island, USA) with $10 \%$ FBS. After $24 \mathrm{~h}$ of co-transfection, the medium was replaced with fresh medium. After $48 \mathrm{~h}$, dual luciferase assay was performed using the Dual 2 Luciferase Assay System (Promega, Madison, USA). Cells were seeded at $5 \times 10^{4}$ cells/75 $\mu \mathrm{l}$ in wells of a 96 well plate and treated with $75 \mu \mathrm{l}$ of luciferase substrate buffer. Luminescence was measured after $10 \mathrm{~min}$ incubation. Relative luciferase activity (RLA) was determined using a luminometer (Turner Biosystems, Sunnyvale, CA, USA) and the transfection efficiencies were normalized according to Renilla activity. All relative luciferase activities were determined by calculating the ratio of firefly and Renilla luciferase activities, and the results are shown as mean \pm standard error of means (SEM; $n=3$ ).

\section{Results}

Notch1 is overexpressed in NPC cell lines. We firstly analyzed the expression level of Notch1 protein in several human cancer cell lines originated from various subsites of head and neck area (oral cavity, hypopharynx and nasopharynx) by western blotting for selection of cell lines for further experiments. Interestingly, two NPC cell lines (HONE1 and HNE1) showed higher levels of Notch1 expression than other HNSCC cell lines (Figure 1A). Thus, we assumed that Notch1 may play an important oncogenic role in NPC cells. To verify this hypothesis, we suppressed the expression of Notch1 gene using a shNotch1 lentiviral construct. Figure 1B shows the successful repression of Notch1 expression in NPC cells, as analyzed by western blotting.

Notch 1 knockdown promotes the in vitro migration and invasion of NPC cells. We evaluated the effect of the inhibition of Notch1 expression on the migration, invasion and wound healing abilities of NPC cells. Unexpectedly, the migra-

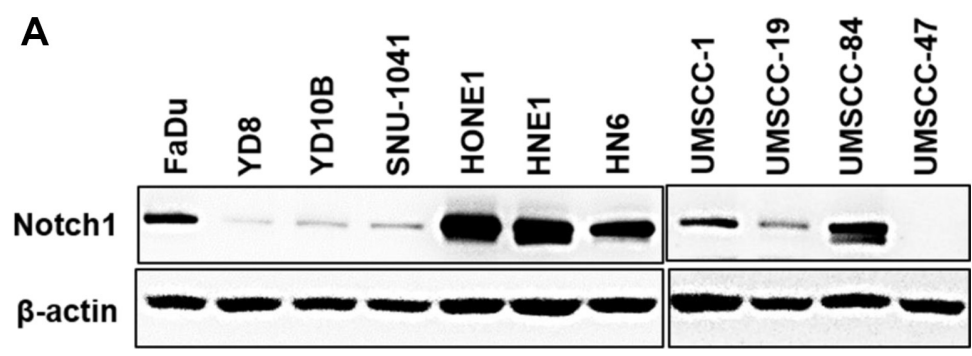

B

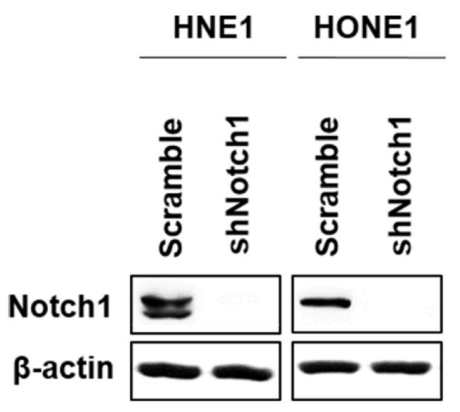

Figure 1. Levels of Notch1 expression in HNSCC cells. A) Notch1 protein expression in various HNSCC cell lines. B) Knockdown of Notch1 expression in NPC cells using either scramble shRNA or shNotch 1 construct. 
tion assay result showed that Notch1 knockdown resulted in an increase in the migration ability of cells as compared with the cells treated with scramble shRNA (Figure 2A). The invasion capacity also significantly increased in these cells subjected to Notch1 knockdown (Figure 2B). Furthermore, Notch1 knockdown resulted in a significant increase in the wound-healing ability of cells as compared with the control cells transfected with the scramble shRNA (Figure 2C). These results show that Notch1 suppression increased the invasion capacity of NPC cells in vitro.

Notch1 knockdown promotes lung metastasis of NPC cells in vivo. One of the causes of treatment failure and death in patients with NPC is the distant metastasis of the cancer cells to the lungs. We investigated the in vivo metastatic effect of Notch1 suppression in NPC cells. We injected NPC cells treated with shNotch1 or scramble vector into the tail veins of mice and found that the number of metastatic nodules in the lungs were significantly increased in the mouse injected with shNotch1 vector-transfected cells as compared with those injected with scramble vector-transfected cells (Figure 3A). Histological analysis also showed the increase in the lung metastasis of the cells transfected with shNotch1 vector as compared with those treated with the scramble vector (Figure 3B). These results confirmed that the knockdown of Notch1 expression in NPC cells promoted the formation of metastatic nodules in the lungs.

Notch1 inactivation increases the expression of Slug gene. EMT plays an important role in cell migration and invasion; therefore, we investigated whether Notch1mediated invasion capacity was induced through EMT activation in NPC cells. Of the three major EMT-related transcriptional factors, such as twist, snail, and slug, Notch1
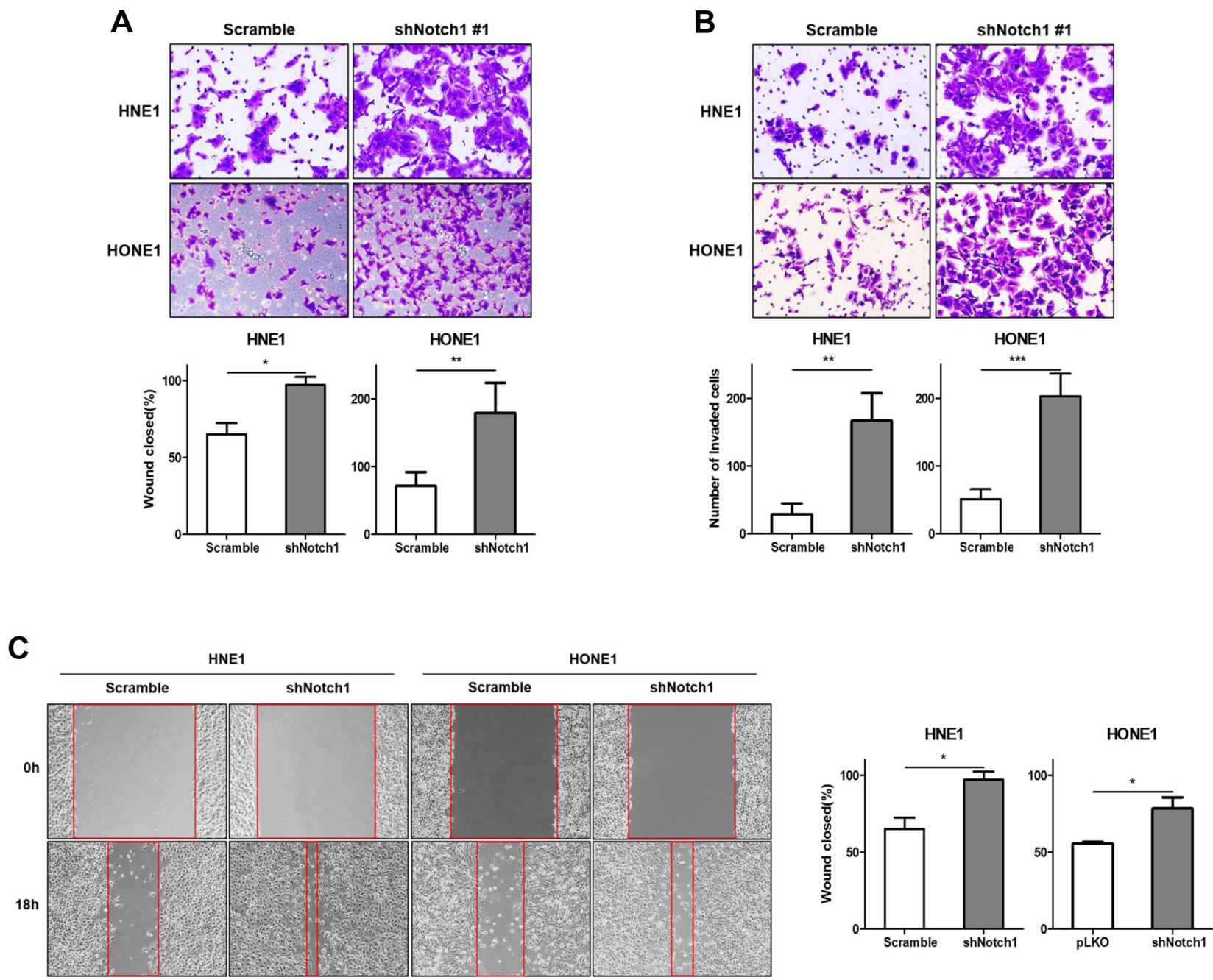

Figure 2. Knockdown of Notch1 expression increases the migration and invasion of NPC cells. A) Migration ability of NPC cells transduced with scramble shRNA or shNotch1. Data represent the means $\pm S D(N=3)\left({ }^{*} p<0.05,{ }^{* *} p<0.01\right)$. B) Invasion ability of NPC cells transduced with scramble shRNA or shNotch1. Data represent the means $\pm \mathrm{SD}(\mathrm{N}=3)\left({ }^{\star} \mathrm{p}<0.05,{ }^{* *} \mathrm{p}<0.01,{ }^{* * *} \mathrm{p}<0.001\right)$. C) Wound-healing ability of NPC cells transduced with scramble siRNA or shNotch1. Data represent the means $\pm \mathrm{SD}(\mathrm{N}=3)\left({ }^{*} \mathrm{p}<0.05,{ }^{* *} \mathrm{p}<0.01\right)$. 
A

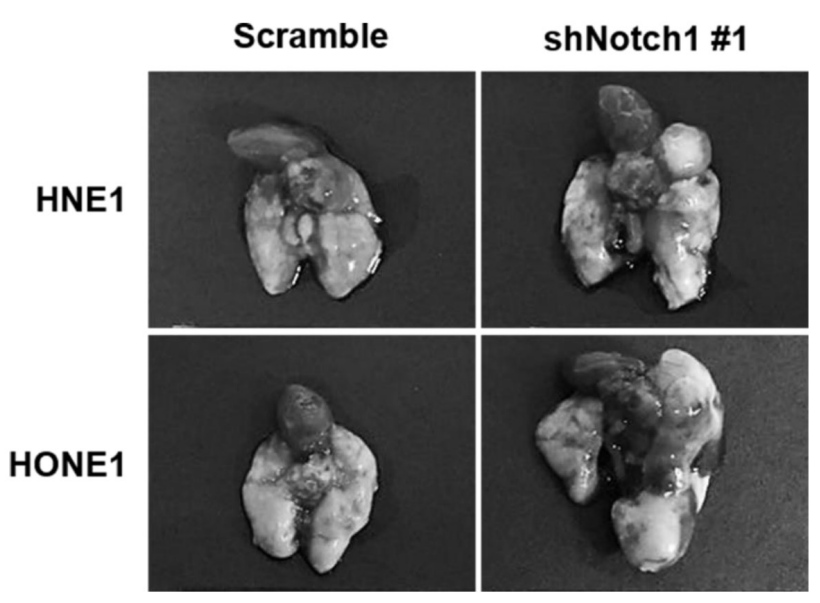

B

Scramble

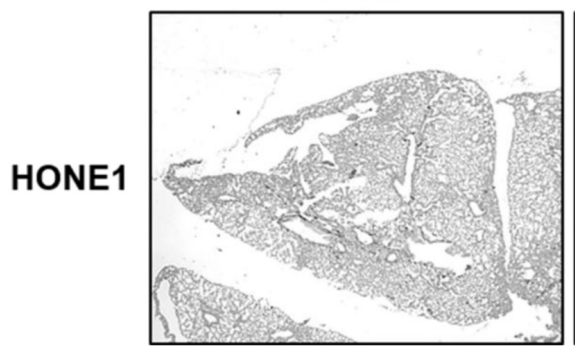

shNotch1 \#1

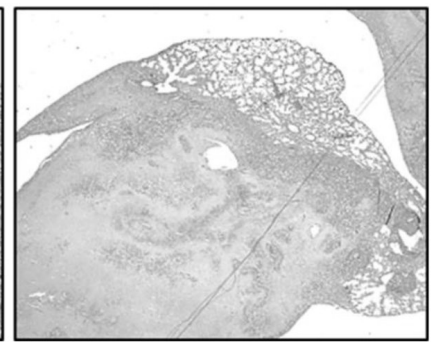

Figure 3. Knockdown of Notch1 expression increases lung metastasis in a mouse model. A) In vivo metastasis of NPC cells transduced with either scramble shRNA or shNotch1 construct. Representative pictures showing tumor in mouse lung. B) Hematoxylin and eosin staining of mouse lung tissue.

suppression significantly increased slug expression at mRNA levels in NPC cells (Figure 4A). Western blot analysis results also confirmed an increase in Slug protein expression following Notch1 knockdown in NPC cells (Figure 4B). However, the expression of MMP, one of major signaling molecules involved in the head and neck cancer invasion ability, showed no change following Notch1 suppression (Figure 4C). To investigate the association between Notch1 and Slug expression, we performed a promoter assay by transfecting NPC cells carrying shNotch1 or scramble vector with the reporter constructs of the pGL3-Slug promoter or its negative control pGL3-basic. shNotch1-transduced NPC cells transfected with Slug promoter showed an increase in luciferase activity as compared to cells transfected with the scramble vector (Figure $4 \mathrm{D}$ ). These results indicate that the knockdown of Notch 1 expression affected the expression of Slug through the activation of Slug promoter at the transcription level.

Notch1 inactivation stimulates the invasion capacity of NPC cells partly through the upregulation of Slug expression. To confirm whether Notch1 inactivation induces cancer cell metastasis through Slug activation, we knocked down the expression of Slug with an siRNA (siSlug) in NPC cells with deleted Notch1 expression and evaluated their migration capacities (Figure 5A). We found that siSlug-transfected cells showed impaired migration and wound-healing capacity as compared with the cells treated with the scramble vector (Figures 5B and 5C). Thus, Notch1 inactivation stimulates the invasion ability of NPC cells partly through the activation of Slug expression.

\section{Discussion}

Notch signaling pathway exerts both oncogenic as well as tumor suppressor roles. The Notch pathway has tumor suppressive roles mainly in squamous epitheliumderived tumors with histological structures similar to those of nasopharynx mucosa, including the esophagus, skin, papillary and bladder cancers. However, studies have also highlighted contradictory results. We have previously reported that Notch1 signaling contributes to the stemness in HNSCC and inactivation of this pathway may serve as a potential approach for the treatment of HNSCC [11]. Yoshida et al. also reported that the altered expression of Notch1 is associated with increased cancer progression and that Notch1 regulates the steps involved in cell metastasis in 
A

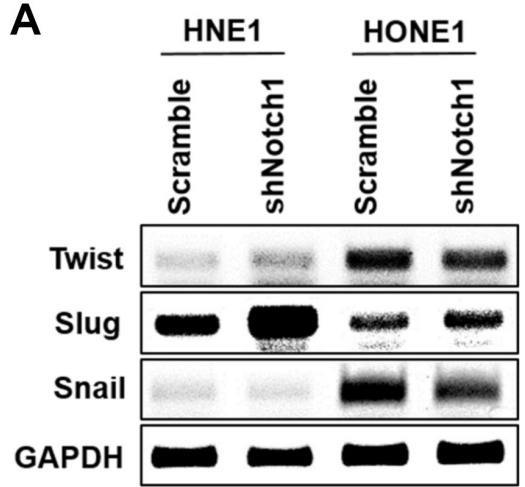

C

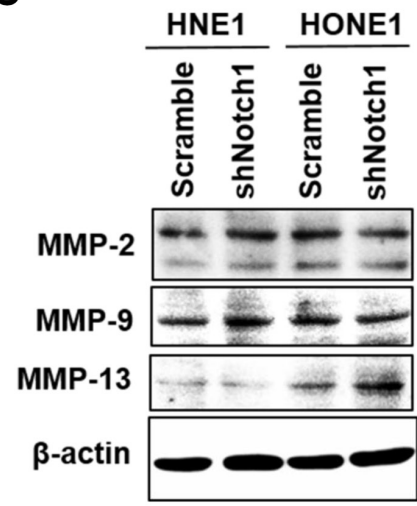

B

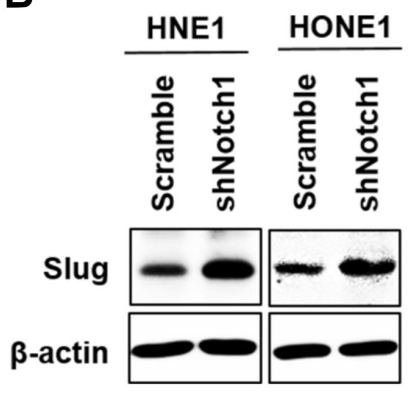

D

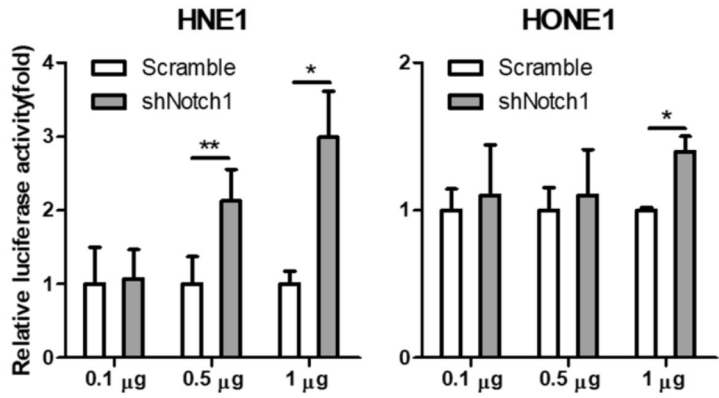

Figure 4. The invasive effects after suppression of Notch 1 expression on NPC metastasis is partly mediated through Slug upregulation. A) mRNA level of EMT markers in NPC cells transduced with either scramble shRNA or shNotch1. B) Protein level of Slug in NPC cells transduced with either scramble shRNA or shNotch1. C) Protein levels of various MMPs in NPC cells transduced with either scramble shRNA or shNotch1. D) Luciferase activity corresponding to Slug expression in NPC cells transduced with either scramble shRNA or shNotch1.

oral SCC [12]. Thus, the role of Notch1 in HNSCC seems to be highly context-dependent.

Zhang et al. found that Notch1 signaling was activated in the cells expressing embryonic stem cell proteins in human primary NPC, suggestive of its role as a regulator of cancerstem like cells in NPC [13]. Yang et al. also observed that the overexpression of Notch1 resulted in an increase in NPC cell growth and invasion in vitro [14]. Thus, Notch1 signaling was thought to contribute to NPC development. However, our present study showed that Notch1 inactivation promotes invasion and metastasis of NPC cells. To the best of our knowledge, this is the first report to demonstrate the suppressive role of Notch1 in NPC cells.

EMT is an event that converts adherent epithelial cells into individual migratory cells, which may invade the extracellular matrix [15]. This process creates cells that act as progenitors of many different tissues during embryonic development [16]. In addition, EMT may contribute to the invasive and metastatic growth of tumors. Thus, it is easy to speculate that EMT-associated cancer cell invasion and the specific factor controlling stem cell phenotype are closely related. Some previous studies have reported that Notch might drive EMT by upregulating the expression of two target factors, Snail and Slug, that serve as transcriptional repressors of $\mathrm{CDH1}$, the gene encoding E-cadherin $[17,18]$. Notch may also synergize with hypoxia-inducible factor-1a (HIF-1 $\alpha$ ) and HIF-2 $\alpha$ to induce EMT and subsequently increase metastasis [19]. In addition, the crosstalk between Notch and transforming growth factor beta (TGF $\beta$ ) is important for the initiation of EMT, as Notch signaling is essential to maintain the TGF $\beta$-induced expression of HEY1 [17]. In the present study, Notch1 activation induced the invasion and metastasis of NPC cells through the upregulation of Slug expression. Leong et al. also observed that the ligand-induced activation of Notch1 promoted tumor growth and metastasis through the induction of Slug expression, characterized with EMT and inhibition of anoikis in breast cancer [20].

In conclusion, our study demonstrates that Notch1 inactivation promotes the metastasis of NPC cells. To date, many 


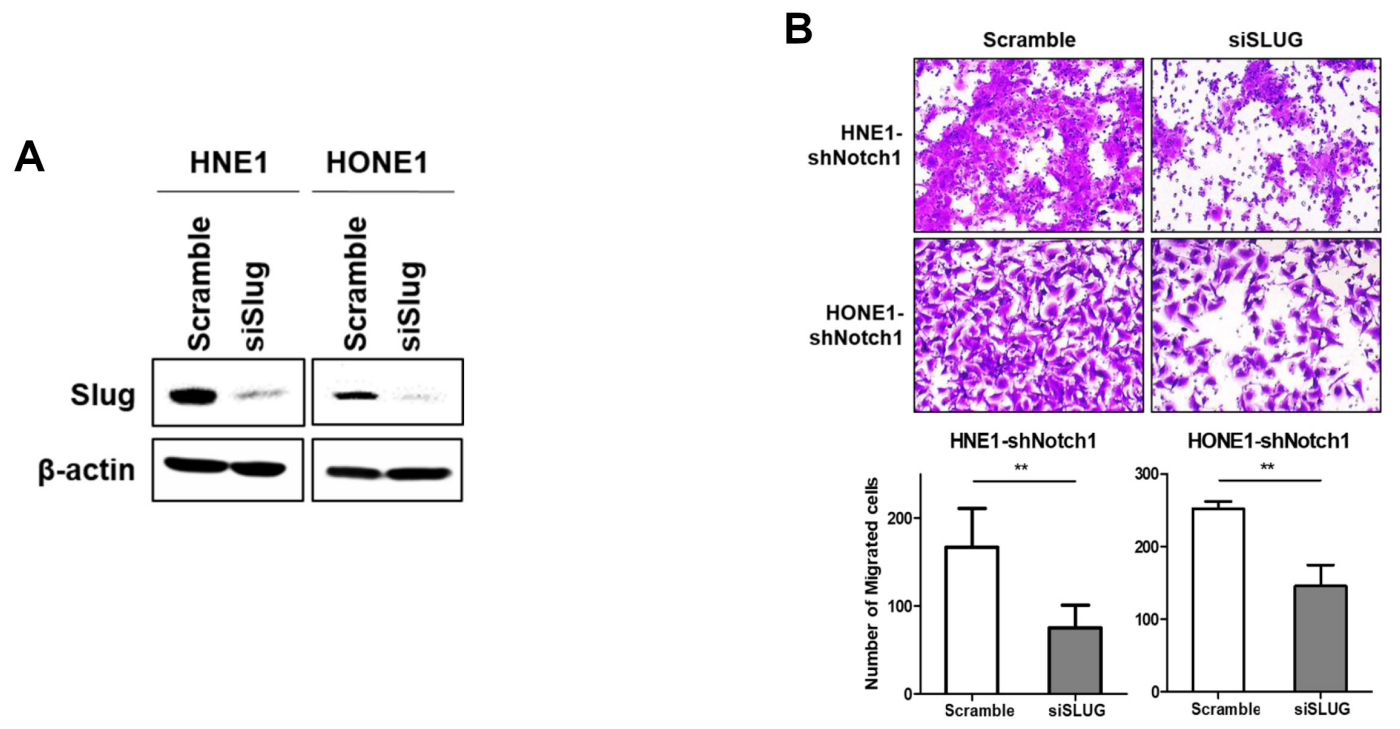

C
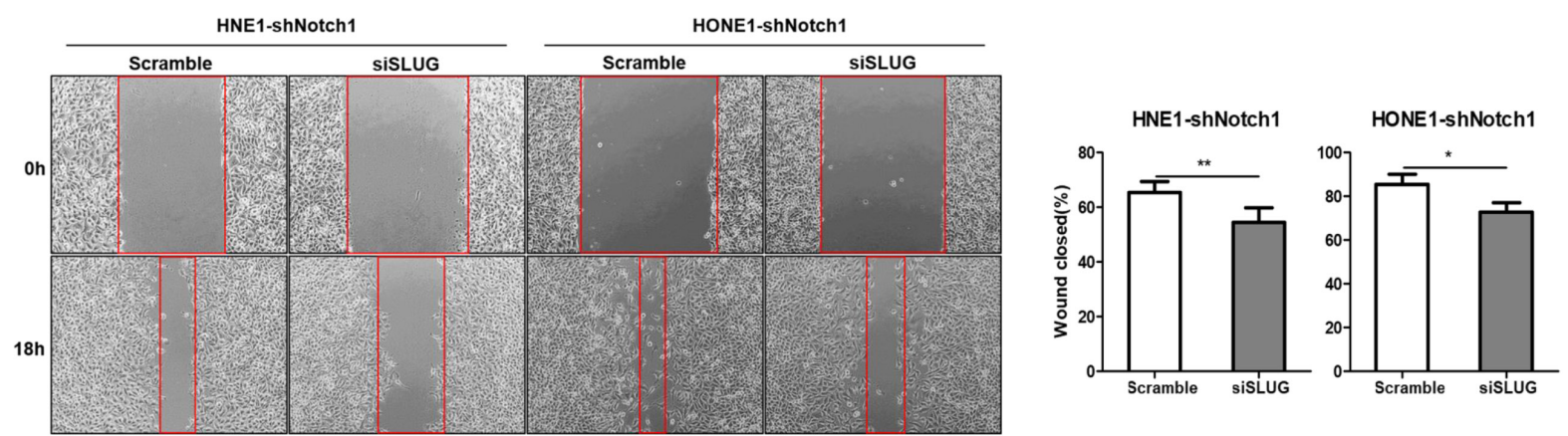

Figure 5. Suppression of Slug expression rescues the metastasis ability of NPC cells transfected with shNotch1. A) Migration ability of shNotch1transfected NPC cells transduced with scramble siRNA or siSlug. Data represent the means $\pm S D(N=3)\left({ }^{\star} p<0.05,{ }^{* *} p<0.01\right)$. B $)$ Wound-healing ability of shNotch1-transfected NPC cells transduced with scramble siRNA or siSlug. Data represent the means $\pm \mathrm{SD}(\mathrm{N}=3)\left({ }^{*} \mathrm{p}<0.05,{ }^{* *} \mathrm{p}<0.01\right)$.

anticancer effects of Notch inhibitors have been reported in different types of cancers, and several drugs have entered into preclinical trials. Our results show that Notch target therapy may increase the invasion and metastasis of NPC, necessitating the prudent use of these agents in clinical trials.

Acknowledgements: This work was supported by Konkuk University Hospital Grant 2016.

\section{References}

[1] WEI WI, SHAM JS. Nasopharyngeal carcinoma. Lancet 2005; 365: 2041-2054. https: //doi.org/10.1016/S01406736(05)66698-6

[2] CHEN W, HU G. Biomarkers for enhancing the radiosensitivity of nasopharyngeal carcinoma. Cancer Biol Med 2015; 12: 23-32. https: //doi.org/10.7497/j.issn.20953941.2014 .0015
[3] LEE AW, LAW SC, FOO W, POON YF, CHEUNG FK et al. Retrospective analysis of patients with nasopharyngeal carcinoma treated during 1976-1985: Survival after local recurrence. Int J Radiat Oncol Biol Phys 1993; 26: 773-782. https: //doi.org/10.1016/0360-3016(93)90491-d

[4] XU L, HUANG T, HU H, WANG MY, SHI SM et al. The developmental transcription factor IRF6 attenuates ABCG2 gene expression and distinctively reverses stemness phenotype in nasopharyngeal carcinoma. Cancer Lett 2018; 431: 230-243. https: //doi.org/10.1016/j.canlet.2017.10.016

[5] MUMM JS, KOPAN R. Notch signaling: from the outside in. Dev Biol 2000; 228: 151-165. https: //doi.org/10.1006/ dbio. 2000.9960

[6] LAI EC. Notch signaling: control of cell communication and cell fate. Development 2004; 131: 965-973. https: //doi. org/10.1242/dev.01074

[7] ARTAVANIS-TSAKONAS S, RAND MD, LAKE RJ. Notch signaling: cell fate control and signal integration in development. Science 1999; 284: 770-776. https: //doi.org/10.1126/ science.284.5415.770 
[8] RANGANATHAN P, WEAVER KL, CAPOBIANCO AJ. Notch signalling in solid tumours: a little bit of everything but not all the time. Nat Rev Cancer 2011; 11: 338-351. https: //doi.org/10.1038/nrc3035

[9] NOWELL CS, RADTKE F. Notch as a tumour suppressor. Nat Rev Cancer 2017; 17: 145-159. https: //doi.org/10.1038/ nrc. 2016.145

[10] BANERJEE D, HERNANDEZ SL, GARCIA A, KANGSAMAKSIN T, SBIROLI E et al. Notch suppresses angiogenesis and progression of hepatic metastases. Cancer Res 2015; 75: 1592-1602. https: //doi.org/10.1158/0008-5472.CAN-141493

[11] LEE SH, DO SI, LEE HJ, KANG HJ, KOO BS et al. Notch1 signaling contributes to stemness in head and neck squamous cell carcinoma. Lab Invest 2016; 96: 508-516. https: // doi.org/10.1038/labinvest.2015.163

[12] YOSHIDA R, NAGATA M, NAKAYAMA H, NIIMORIKITA K, HASSAN W et al. The pathological significance of Notch1 in oral squamous cell carcinoma. Lab Invest 2013; 93: 1068-1081. https: //doi.org/10.1038/labinvest.2013.95

[13] ZHANG Y, PENG J, ZHANG H, ZHU Y, WAN L et al. Notch1 signaling is activated in cells expressing embryonic stem cell proteins in human primary nasopharyngeal carcinoma. J Otolaryngol Head Neck Surg 2010; 39: 157-166.

[14] YANG X, NI W, LEI K. miR-200b suppresses cell growth, migration and invasion by targeting Notch 1 in nasopharyngeal carcinoma. Cell Physiol Biochem 2013; 32: 1288-1298. https: //doi.org/10.1159/000354527
[15] ACLOQUE H, ADAMS MS, FISHWICK K, BRONNERFRASER M, NIETO MA. Epithelial-mesenchymal transitions: the importance of changing cell state in development and disease. J Clin Invest 2009; 119: 1438-49. https: //doi. org/10.1172/JCI38019

[16] PUISIEUX A, BRABLETZ T, CARAMEL J. Oncogenic roles of EMT-inducing transcription factors. Nat Cell Biol 2014; 16: 488-494. https: //doi.org/10.1038/ncb2976

[17] ZHOU J, JAIN S, AZAD AK, XU X, YUN HC et al. Notch and TGF $\beta$ form a positive regulatory loop and regulate EMT in epithelial ovarian cancer cells. Cell Signal 2016; 28: 838849. https: //doi.org/10.1016/j.cellsig.2016.03.016

[18] ZHOU P, WANG C, HU Z, CHEN W, QI W et al. Genistein induces apoptosis of colon cancer cells by reversal of epithelial-to-mesenchymal via a Notch1/NF- $\mathrm{B} / \mathrm{slug} / \mathrm{E}-$ cadherin pathway. BMC Cancer 2017; 17: 813. https: //doi. org/10.1186/s12885-017-3829-9

[19] SAHLGREN C, GUSTAFSSON MV, JIN S, POELLINGER L, LENDAHL U. Notch signaling mediates hypoxia-induced tumor cell migration and invasion. Proc Natl Acad Sci U S A 2008; 105: 6392-6397. https: //doi.org/10.1073/ pnas.0802047105

[20] LEONG KG, NIESSEN K, KULIC I, RAOUF A, EAVES C, POLLET I, et al. Jagged1-mediated Notch activation induces epithelial-to-mesenchymal transition through Slug-induced repression of E-cadherin. J Exp Med 2007; 204: 2935-2948. https: //doi.org/10.1084/jem.20071082 